\title{
Resource Allocation and Relay Selection for Multi-User OFDM-Based Cooperative Networks with SWIPT
}

\author{
Sumit Gautam, Eva Lagunas, Symeon Chatzinotas and Björn Ottersten \\ Interdisciplinary Centre for Security, Reliability and Trust (SnT) \\ University of Luxembourg, 29, avenue JF Kennedy, L-1855 Luxembourg \\ Email: \{sumit.gautam,eva.lagunas,symeon.chatzinotas,bjorn.ottersten\}@uni.lu
}

\begin{abstract}
In this paper, we investigate resource allocation and relay selection in a two-hop relay-assisted multi-user network, where the end users support Simultaneous Wireless Information and Power Transfer (SWIPT). In particular, we consider singlesource assisted by a set of spatially distributed relays able to amplify-and-forward orthogonal frequency division multiplexing (OFDM) signals carrying both data and power at the same time. The users are assumed to implement a power splitting technique where the received signal is orthogonally split in two streams of different power levels, where one signal stream is sent to the power harvesting module while the other is converted to baseband for information decoding. We aim at optimizing the users' power splitting ratios as well as the relay, carrier and power assignment so that the end-users' sum-rate is maximized subject to transmitted and harvested power constraints. Such joint optimization is combinatorial in nature with a non-convex structure. Therefore, we present a sub-optimal low complex solution based on the harmonic mean of the two-hop relay channel coefficients. Simulation results reveal that the proposed algorithm provides significant performance gains in comparison with a semi-random resource allocation and relay selection approach.
\end{abstract}

\section{INTRODUCTION}

As the volume of data traffic grows, energy-efficient network design has become a great concern [1]. The reason is three-fold: First, to reduce the worldwide electric energy consumption and achieve eco-sustainable telecommunication networks; second, to reduce the associated energy cost; and third, to prolong the battery life of battery-powered wireless devices. Therefore, energy efficiency is of crucial importance in future wireless communication [2].

Recently, the concept of Simultaneous Wireless Information and Power Transfer (SWIPT) has been introduced as a promising approach to reduce the energy consumption while satisfying the required Quality-of-Service (QoS) [3][5]. Thanks to the recent advances in hardware technologies [6], SWIPT allows the receivers not only to receive data from the transmitter, but also to recycle the received signal power in order to reduce their own power consumption. In this paper, we focus on a SWIPT Power Splitting (PS) receiver architecture [7], where the received signal is splitted into two streams of different power in order to decode information and harvest energy separately, yet simultaneously. This kind of receiver architecture has been shown to achieve better rate-energy (R-
E) transmission trade-off compared to the time-splitting alternative, at an expense of a more complex switching circuitry [4].

On the other hand, cooperative relaying has emerged as a promising technique to improve the coverage and overall throughput [8]. Adoption of relaying technology can offer substantial benefits in multi-user multi-carrier systems, where different sub-carriers experience different channels for different users, particularly in scenarios where the sourcedestination pairs are located far apart. Moreover, cooperative communications can provide additional advantage to improve the SWIPT performance [9]. In particular, the power transfer distance is largely limited by the power sensitivity of the energy harvester, which considering the current state-of-theart technology is of $-10 \mathrm{dBm}$, significantly tighter than the $-60 \mathrm{dBm}$ assumed for effective information receivers [10].

In this paper, we focus on the Amplify-and-Forward (AF) relay protocol, where relays simply forward the received signal after amplification. Furthermore, we assume a multi-user Orthogonal Frequency Division Multiple Access (OFDMA) system with orthogonal user multiplexing. Orthogonal Frequency Division Multiplexing (OFDM) has become the key physicallayer technique in modern wireless communication systems due to its robustness against the frequency selectivity of wireless channels [11], [12]. Furthermore, OFDM is very attractive from a multi-user perspective, since different carriers can be assigned to various users depending on their throughput demands. In the considered OFDM-based cooperative scenario, intelligent resource allocation is of paramount importance in order to make the most out of the overall available resources.

There have been numerous works considering OFDM resource allocation both in conventional and relay-aided communication networks. Focusing on the relay-aided works, [13], [14] have addressed the joint power and sub-carrier allocation, where it is shown that the number of OFDM sub-carriers assigned to each relay can be flexibly configured according to the channel state information so that the source-destination link rate is maximized. Several works have investigated the relay selection in two-hop systems [15], [16], where a common selection strategy is to choose the relay with best equivalent end-to-end channel gain. The above-mentioned works, however, have not considered relay selection together with 
IEEE $15^{\text {th }}$ Int. Symp. on Wireless Comm. Systems 2018 (ISWCS'18), Lisbon, Portugal

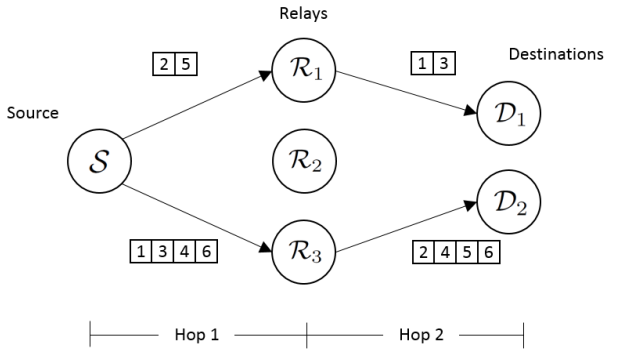

Fig. 1: Multi-relay multi-user OFDM-based system model with $K=3$ and $L=2$.

transmitted power and sub-carrier assignment. Joint allocation of these three resources is studied in [17], [18] for a single OFDM source-destination link. The benefits of cooperative transmission for OFDM-SWIPT are much less investigated [19], [20]. Recently, relay selection along with the design of the optimal SWIPT splitting factor has been addressed in [21] for a two-hop relaying network considering power harvesting constraints at the receiver.

In contrast to the above studies, this paper investigates the single relay selection from a pool of candidate relays, subcarrier pairing, power allocation and PS ratio optimization in a two-hop relay-assisted multi-user network with SWIPT. Single relay is chosen over multiple relays to significantly reduce the control and synchronization process among the relay nodes. We formulate the resource allocation and relay selection problem as a maximization of the total system throughput by satisfying the individual users' energy harvesting constraints subject to transmit power limits. The latter is an extremely challenging problem due to the complexity caused by the joint optimization of several network resources, which requires an analysis within the full search space. In order to circumvent this tedious and unaffordable optimization, we follow an heuristic approach based on the equivalent end-to-end channel gain computed with the harmonic mean as in [15], [16], [18]. Numerical results are presented, which show that the proposed low complexity scheme is able to offer better performance than the one achieved with a semi-random resource assignment approach, where the relay and sub-carriers are randomly assigned followed by a optimal power and PS ratios allocation according to the previous random decisions.

The remainder of this paper is organized as follows. Section II introduces the system model. The problem formulation for the optimal solution is presented in Section III. The proposed sub-optimal scheme is described in Section IV. Numerical results are shown in Section $\mathrm{V}$, followed by concluding remarks in Section VI.

\section{System ModeL}

Depicted in Fig. 1, the system model consists of single source $\mathcal{S}$, that transfers information and power to $L$ destinations $\left(\mathcal{D}_{1}, \ldots, \mathcal{D}_{\ell}\right)$ via a set of $K$ non-regenerative half-duplex relays $\left(\mathcal{R}_{1}, \ldots, \mathcal{R}_{K}\right)$. In general, $L \leq K$. Source, relay and destination nodes are equipped with single antenna. The direct source-destination link is assumed to suffer strong attenuation and thus it is neglected in the system model. The communication is performed in two orthogonal time slots. In particular,

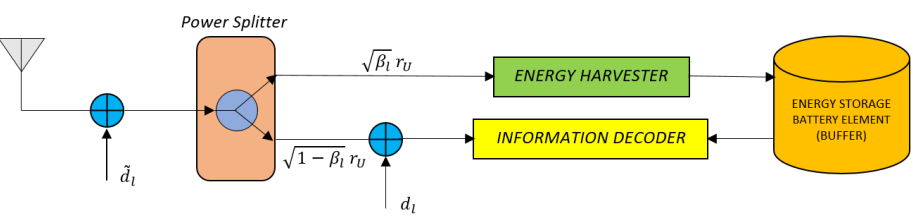

Fig. 2: User receiver architecture based on PS scheme.

during the first time slot, the source transmits the signal to the selected set of relay nodes. In the second time slot, the selected relays forward an amplified version of the received signal to the intended destination. Each destination is assisted by single relay, which is not shared with other destinations. The transmission is based on OFDM with $N$ orthogonal flatfading sub-carriers referred to as $1 \leq n \leq N$ for the first hop and $1 \leq n^{\prime} \leq N$ for the second hop. We consider a sub-carrier pairing approach, where the relay amplifies-and-forwards on a sub-carrier pair basis, by receiving from sub-carrier $n$ and transmitting on sub-carrier $n^{\prime}$, thus forming a sub-carrier pair $\left(n, n^{\prime}\right)$. Note that $n$ and $n^{\prime}$ may be equal or not. Similar to [17], we assume that each sub-carrier pair can only be assigned to one relay but multiple sub-carrier pairs can be assigned to a single relay. An example of relay selection and OFDM sub-carrier pairing for a multi-relay multi-user network with $N=6, K=3$ and $L=2$ is illustrated in Fig. 1 .

The channel coefficient of the first hop between $\mathcal{S}$ and $\mathcal{R}_{k}$ on the $n$-th sub-carrier is denoted as $h_{1, n, k}$, whereas the channel coefficient of the second hop between the $\mathcal{R}_{k}$ and $\mathcal{D}_{\ell}$ on the $n^{\prime}$-th sub-carrier is denoted as $h_{2, n^{\prime}, k, \ell}$. The transmit power on the $n$-th sub-carrier at the source $\mathcal{S}$ in the first hop is denoted as $p_{1, n}$, while the $\mathcal{R}_{k}$ retransmits the received signal by applying the following amplification coefficient

$$
g_{\left(n, n^{\prime}\right), k}=\sqrt{\frac{p_{2, n^{\prime}, k}}{p_{1, n}\left|h_{1, n, k}\right|^{2}+\sigma_{k}^{2}}}
$$

which ensures that the $k$-th relay transmits with a power $p_{2, n^{\prime}, k}$ on the $n^{\prime}$-th sub-carrier. In (1), $\sigma_{k}^{2}$ denotes the noise power at the $\mathcal{R}_{k}$ relay. The total available power at the source $\mathcal{S}$ and at the relay $\mathcal{R}_{k}$ is fixed as $P_{S}$ and $P_{\mathcal{R}, k}$, respectively.

The received signal at the destination $\mathcal{D}_{\ell}$ is split into two streams according to a PS ratio $\beta_{\ell}$, as shown in Fig. 2. A fraction $\sqrt{\beta_{\ell}}$ of the received signal power is used for energy harvesting, while the remaining is sent to the information decoder. For the sake of simplicity, we assume a normalized transmission time in each hop so that the terms energy and power can be used interchangeably. In practice, the antenna noise $\tilde{d}_{\ell} \in \mathcal{C N}\left(0, \sigma_{\tilde{d}_{\ell}}^{2}\right)$ has a negligible impact on both the information receiving and energy harvesting, since $\sigma_{\tilde{d}_{f}}^{2}$ is generally much smaller than the noise power introduced by the baseband processing circuit, and thus even lower than the average power of the received signal [7]. Correspondingly, we ignore the noise term $\tilde{d}_{\ell}$ in the following analysis. In this paper, the relay nodes are assumed to have their own power supply and, therefore, they do not need to harvest energy from the received signals.

The effective signal to noise ratio (SNR) seen at the decoding branch of the destination $\mathcal{D}_{\ell}$ for the $\left(n, n^{\prime}\right)$ sub-carrier pair over the $\mathcal{S} \rightarrow \mathcal{R}_{k} \rightarrow \mathcal{D}_{\ell}$ link is given by, 


$$
\gamma_{\left(n, n^{\prime}\right), k, \ell}=\frac{\left(1-\beta_{\ell}\right) p_{1, n}\left|h_{1, n, k} g_{\left(n, n^{\prime}\right), k} h_{2, n^{\prime}, k, \ell}\right|^{2}}{\left(1-\beta_{\ell}\right) \sigma_{k}^{2}\left|h_{2, n^{\prime}, k, \ell} g_{\left(n, n^{\prime}\right), k}\right|^{2}+\sigma_{d_{\ell}}^{2}},
$$

where $\sigma_{d_{\ell}}^{2}$ denotes the noise power introduced by the downconversion procedure at $\mathcal{D}_{\ell}$. The above expression can be simplified and re-written as follows

$$
\gamma_{\left(n, n^{\prime}\right), k, \ell}=\frac{\left(1-\beta_{\ell}\right) \gamma_{1, n, k} \gamma_{2, n^{\prime}, k, \ell}}{1+\gamma_{1, n, k}+\left(1-\beta_{\ell}\right) \gamma_{2, n^{\prime}, k, \ell}},
$$

where $\gamma_{1, n, k}=\frac{p_{1, n}\left|h_{1, n, k}\right|^{2}}{\sigma_{k}^{2}}$, and $\gamma_{2, n^{\prime}, k, \ell}=\frac{p_{2, n^{\prime}, k}\left|h_{2, n^{\prime}, k, \ell}\right|^{2}}{\sigma_{d_{\ell}}^{2}}$. Assuming that the SNR of the relayed signal is high, we can further simplify the expression by applying the following approximation,

$$
\hat{\gamma}_{\left(n, n^{\prime}\right), k, \ell} \approx \frac{\left(1-\beta_{\ell}\right) \gamma_{1, n, k} \gamma_{2, n^{\prime}, k, \ell}}{\gamma_{1, n, k}+\left(1-\beta_{\ell}\right) \gamma_{2, n^{\prime}, k, \ell}} .
$$

The aforementioned approximation is commonly used in the literature to make the problem more tractable [13], [17].

The throughput achieved by the decoding branch of the $\left(n, n^{\prime}\right)$ sub-carrier pair over the $\mathcal{S} \rightarrow \mathcal{R}_{k} \rightarrow \mathcal{D}_{\ell}$ link can thus be expressed as

$$
R_{\left(n, n^{\prime}\right), k, \ell}=\frac{1}{2} \ln \left(1+\hat{\gamma}_{\left(n, n^{\prime}\right), k, \ell}\right),
$$

where the factor $1 / 2$ is introduced to compensate for the two time slots of the considered relay assisted communication.

On the other hand, the energy harvested at the harvesting branch for the $\left(n, n^{\prime}\right)$ sub-carrier pair over the $\mathcal{S} \rightarrow \mathcal{R}_{k} \rightarrow \mathcal{D}_{\ell}$ link is given by

$$
E_{\left(n, n^{\prime}\right), k, \ell}=\zeta \cdot \beta_{\ell} \cdot\left(\left|g_{k} h_{2, n^{\prime}, k, \ell}\right|^{2}\left(p_{1, n}\left|h_{1, n, k}\right|^{2}+\sigma_{k}^{2}\right)\right),
$$

which can be simplified by using the expression in (1), resulting in,

$$
E_{\left(n, n^{\prime}\right), k, \ell}=\zeta \cdot \beta_{\ell} \cdot p_{2, n^{\prime}, k}\left|h_{2, n^{\prime}, k, \ell}\right|^{2},
$$

where $\zeta$ is the energy conversion efficiency of the receiver.

\section{PROBlem Formulation}

Our goal is to design the sub-carrier pairing, relay selection, power allocation and PS ratio optimization so that the total end-to-end user sum-rate is maximized subject to a set of transmitted and harvested power constraints.

Let us denote $\phi_{\left(n, n^{\prime}\right)} \in\{0,1\}$ as the indicator for subcarrier pairing, where $\phi_{\left(n, n^{\prime}\right)}=1$ means that sub-carrier $n$ in the first hop is paired with sub-carrier $n^{\prime}$ of the second hop and $\phi_{\left(n, n^{\prime}\right)}=0$ otherwise. Each sub-carrier in the first hop can be paired with one and only one sub-carrier of the second hop. Therefore, the binary variable $\phi_{\left(n, n^{\prime}\right)}$ must satisfy the following sub-carrier pairing constraint,

$$
\sum_{n=1}^{N} \phi_{\left(n, n^{\prime}\right)}=1, \forall n^{\prime} ; \quad \sum_{n^{\prime}=1}^{N} \phi_{\left(n, n^{\prime}\right)}=1, \forall n .
$$

For the relay selection problem, we make use of the binary variable $s_{k, \ell}=\{0,1\}$, where $s_{k, \ell}=1$ means that $\mathcal{R}_{k}$ is selected for $\mathcal{D}_{\ell}$. It is clear that one relay will be allocated to one and only one user, therefore the following relaydestination constraints must be satisfied,

$$
\sum_{k=1}^{K} s_{k, \ell}=1, \forall \ell ; \quad \sum_{\ell=1}^{L} s_{k, \ell} \leq 1, \forall k .
$$

In order to link each sub-carrier pairs with the corresponding destination node, we define $a_{n, \ell} \in\{0,1\}$ as the binary variable with $a_{n, \ell}=1$ indicating that sub-carrier $n$ is used in the first hop for reaching destination $\mathcal{D}_{\ell}$, and $a_{n, \ell}=0$ otherwise. The destination assignment constraint employs the following assignment rule

$$
\sum_{\ell=1}^{L} a_{n, \ell}=1, \quad \forall n .
$$

Using the previous variable definitions together with (5), we formulate the rate achieved at $\mathcal{D}_{\ell}$ as follows,

$$
R_{\ell}=\sum_{i=1}^{L} \sum_{k=1}^{K} \sum_{n=1}^{N} \sum_{n^{\prime}=1}^{N} a_{n, \ell} \phi_{\left(n, n^{\prime}\right)} s_{k, \ell} R_{\left(n, n^{\prime}\right), k, i} .
$$

Regarding the power constraints, we assume

$$
\begin{gathered}
\sum_{n=1}^{N} p_{1, n} \leq P_{S}, \\
\sum_{n^{\prime}=1}^{N} \sum_{\ell=1}^{L} \sum_{n=1}^{N} s_{k, \ell} a_{n, \ell} \phi_{\left(n, n^{\prime}\right)} p_{2, n^{\prime}, k} \leq P_{R, k}, \quad k=1, \cdots, K,
\end{gathered}
$$

where (C4) and (C5) represents the source power constraint and the relay power constraints, respectively.

On the other hand, the overall energy harvested at $\mathcal{D}_{\ell}$ considering all the intended sub-carriers is given by,

$$
E_{\ell}=\sum_{k=1}^{K} \sum_{i=1}^{L} \sum_{n=1}^{N} \sum_{n^{\prime}=1}^{N} s_{k, \ell} \phi_{\left(n, n^{\prime}\right)} E_{\left(n, n^{\prime}\right), k, i} .
$$

As a consequence, the minimum harvested power constraint at destination $\mathcal{D}_{\ell}$ can be stated as follows,

$$
E_{\ell} \geq \eta_{\ell}, \quad \ell=1, \cdots, L,
$$

where $\eta_{\ell}$ is the minimum harvested power demanded by the user $\mathcal{D}_{\ell}$.

Therefore, the proposed optimization problem can mathematically be represented as follows,

$$
\begin{aligned}
(P 1): \max _{\{\boldsymbol{\phi}, \mathbf{s}, \mathbf{a}, \mathbf{p}, \boldsymbol{\beta}\}} & \sum_{\ell=1}^{L} R_{\ell} \\
\text { subject to : } & (C 1),(C 2),(C 3), \\
& (C 4),(C 5),(C 6), \\
& (C 7): 0 \leq \beta_{\ell} \leq 1, \ell=1, \cdots, L,(13)
\end{aligned}
$$

where $\phi=\left\{\phi_{\left(n, n^{\prime}\right)}\right\}, \mathbf{s}=\left\{s_{k, \ell}\right\}, \mathbf{a}=\left\{a_{n, \ell}\right\}, \mathbf{p}=$ $\left\{p_{1, n}, p_{2, n^{\prime}, k}\right\}$ and $\boldsymbol{\beta}=\left\{\beta_{\ell}\right\}$ denote the optimization variables corresponding to sub-carrier pairing, relay selection, carrierdestination assignment, power allocation and PS ratio, respectively. In the following, we assume that (P1) is feasible and thus an optimal solution exists. However, the problem (P1), unfortunately, is very difficult to solve due to the lack of convexity and due to the complexity caused by joint optimization of resource allocation variables. Therefore, we propose a sub- 


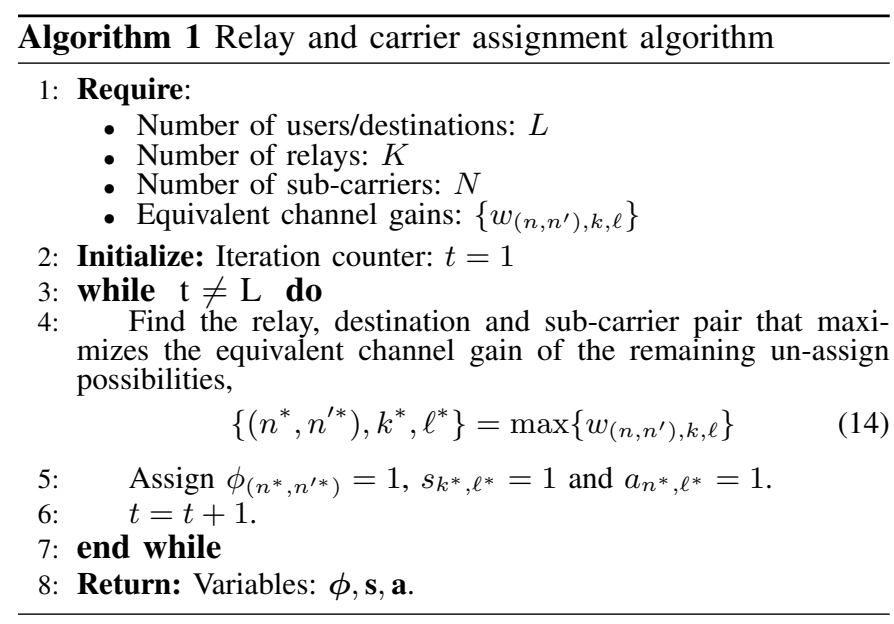

optimal approach to deal with the optimization problem in (P1). Herein, we assume that the source node has full Channel State Information (CSI). We consider that the source node solves the optimization problem and, subsequently, it forwards instructions to the other nodes through proper signaling.

\section{Proposed Sub-Optimal Resource Allocation}

The proposed solution is divided into three steps: First, the relay selection, sub-carrier pairing and carrier-destination variables $(\phi, \mathbf{s}, \mathbf{a})$ are jointly determined based on the harmonic mean metric of the two-hop channel coefficients. Next, the transmitted powers (p) are optimized based on conventional Water-Filling (WF) over the assigned links. Finally, the power splitting ratios $(\boldsymbol{\beta})$ are assigned so that the set of constraints in (C6) are satisfied. Each step is described in detail in the following sections.

\section{A. Relay selection and sub-carrier assignment}

The end-to-end performance of two-hop non-regenerative systems is linked to the harmonic mean of the SNRs of the two hops [16]. Inspired by this, the maximum-harmonicmean relay selection scheme has been proposed in the literature, where the relay with maximum harmonic mean of the two channel gains is selected [15], [18]. Let us define the equivalent channel for the $\left(n, n^{\prime}\right)$ sub-carrier pair over the $\mathcal{S} \rightarrow \mathcal{R}_{k} \rightarrow \mathcal{D}_{\ell}$ link as the harmonic mean of the two-channelhops as follows,

$$
w_{\left(n, n^{\prime}\right), k, \ell}=\frac{2 h_{1, n, k} \cdot h_{2, n^{\prime}, k, \ell}}{h_{1, n, k}+h_{2, n^{\prime}, k, \ell}} .
$$

In this paper, we propose a sub-optimal relay and sub-carrier assignment method where we sequentially assign the relay and sub-carrier pair that maximizes the equivalent channel metric in (15). The corresponding algorithm is summarized in Algorithm 1. Note that Algorithm 1 returns the relay assignment variable $\mathbf{s}$ but an incomplete version of the carrier related variables $\phi$ and a, since only the sub-carrier pairs associated with the assigned relays have been determined. In order to fully determine the sub-carrier pairs and assign them to the corresponding destination, we propose a similar approach where the remaining equivalent channel coefficients

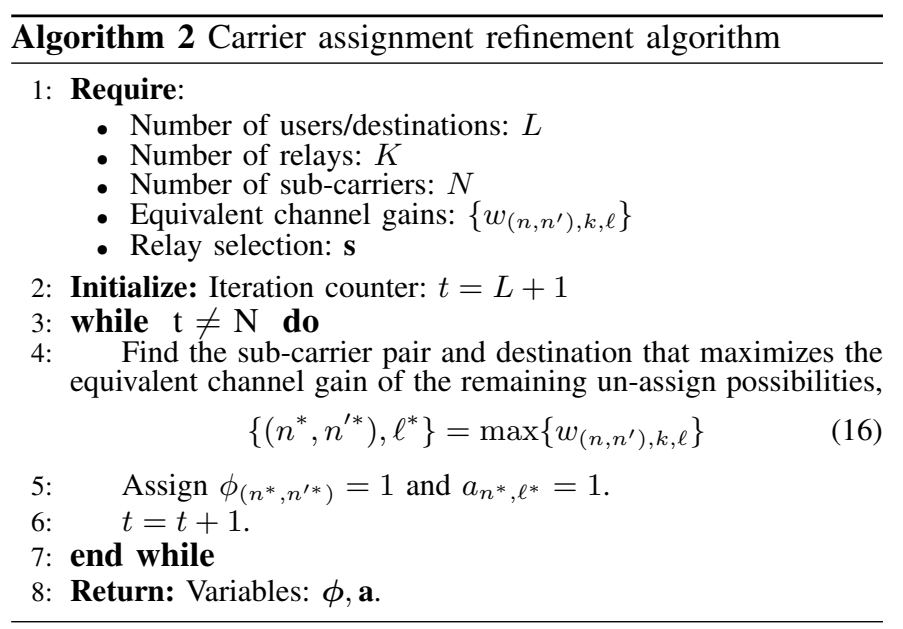

$\left\{w_{\left(n, n^{\prime}\right), k, \ell}\right\}$ are sequentially maximized, taking into account the previous relay selection. The carrier refinement algorithm is summarized in Algorithm 2.

It is noteworthy that the proposed harmonic-mean based approach has a far lesser time complexity of $\mathcal{O}\left(L N^{3}\right)$ in comparison to an optimal exhaustive search approach with $(K \cdot L)^{N !}$ possible combinations of $\phi, \mathbf{s}$, and a. The exhaustive search method becomes inconvenient for higher values of $K, L$, and $N$, due to extremely high time-complexity. However, the time-complexity of the random selection method is very fast and of $\mathcal{O}\left(N^{2}\right)$, but using this kind of hit-and-trial technique has the probability of getting the optimal choice as $1 /(K \cdot L)^{N !}$. Therefore, the random selection method is considered to provide a sub-optimal solution unless the hitand-trial method coincides with optimal selection.

\section{B. Power allocation}

Once the relay and sub-carriers have been assigned, the transmitted power $\mathbf{p}=\left\{p_{1, n}, p_{2, n^{\prime}, k}\right\}$ can be efficiently assigned according to the channel conditions by using the wellknown WF strategy. In particular, we consider a WF approach for the source and for each of the active relay nodes. Therefore, the transmitted power on the first hop is given by,

$$
p_{1, n}= \begin{cases}\frac{1}{\nu}-\varphi_{1, n}, & \text { if } \nu<\frac{1}{\varphi_{1, n}} \\ 0, & \text { otherwise, }\end{cases}
$$

where $\varphi_{1, n}=\frac{\sigma_{k}^{2}}{h_{1, n, k}}$ acts as an indicator of the channel condition between the source and the $k$-th relay over subcarrier $n$ and $\nu$ is the decision threshold.

Similarly, the transmitted power on the second hop for the $k$-th relay can be determined as,

$$
p_{2, n^{\prime}, k}= \begin{cases}\frac{1}{\nu}-\varphi_{2, n^{\prime}, k}, & \text { if } \nu<\frac{1}{\varphi_{2, n^{\prime}, k}} \\ 0, & \text { otherwise, }\end{cases}
$$

where $\varphi_{2, n^{\prime}, k}=\frac{\sigma_{\ell}^{2}}{h_{2, n^{\prime}, k, \ell}}$ acts as an indicator of the channel condition between the $k$-th relay and the $\ell$-th destination over sub-carrier $n^{\prime}$.

\section{Computation of power splitting (PS) ratios}

In this section, we address the computations of optimal PS ratios $\boldsymbol{\beta}=\left\{\beta_{\ell}\right\}$. The proposed solution is found by letting 
IEEE $15^{\text {th }}$ Int. Symp. on Wireless Comm. Systems 2018 (ISWCS'18), Lisbon, Portugal

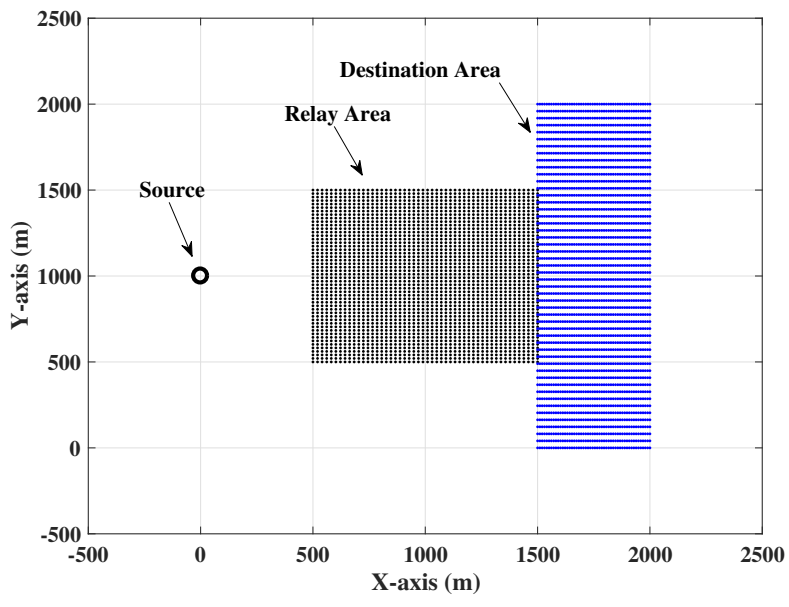

Fig. 3: Simulated scenario

equality hold for the set of constraints in (C6). Therefore, the optimal values $\beta_{\ell}^{*}$ are given by,

$$
\beta_{\ell}^{*}=\frac{\eta_{\ell}}{E_{\ell, \max }},
$$

where $E_{\ell, \max }$ is computed with (9) assuming $\beta_{\ell}=1$.

\section{Simulation Results}

In this section, we present some simulation results to evaluate the performance of the proposed resource allocation and relay selection strategy. We consider the node distribution shown in Fig. 3, where the $K$ relay nodes are placed randomly within a $1 \mathrm{Km}^{2}$ region between the source and the destination area. The destination nodes are placed randomly as well within a $2 \times 0.5 \mathrm{Km}^{2}$ area. We consider frequency-selective channel model, with $M=3$ time-domain taps subject to Rayleigh fading and path-loss, with a path loss exponent equal to $\alpha=2$. More precisely, the time-domain taps are distributed as,

$$
\mathcal{C N} \sim\left(0, \frac{1}{M(1+\hat{d})^{\alpha}}\right),
$$

where $\hat{d}$ is the distance between transmitter and receiver in meters. The corresponding frequency domain channel coefficients are given by the Fourier Transformation with $N$ sub-carriers. For simplicity, we assume that the source and all the relays are subject to the same power constraint $P_{S}=P_{R, 1}=P_{R, 2}=$ $\ldots=P_{R, K}$. The noise power at the relay and destination nodes is also assumed the same and equal to $-100 \mathrm{dBW}$.

For comparison purposes, we consider a random relay and sub-carrier assignment, followed by the proposed power allocation and power splitting assignment as in section IV-B and IV-C, respectively. This comparison method is chosen due to lack of prior work in the specific problem. Fig. 4 compares the Cumulative Density Function (CDF) of the achieved sum-rate after 128 realizations of the proposed and the random strategy in a network composed of $K=3$ relays and $L=2$ users, with $N=6$ OFDM sub-carriers and maximum transmitted power of $5 \mathrm{~W}$. At each realization, different channel coefficients and different locations for the relay and destination nodes are selected. The demanded power $\eta_{\ell}$ is set to $3 \mu \mathrm{W}$ for all the destination nodes. We observe that the proposed resource

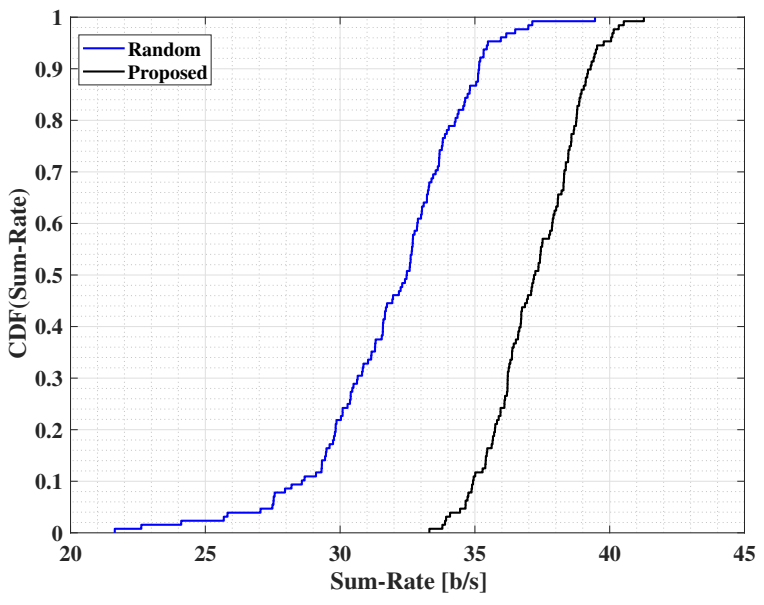

Fig. 4: $\mathrm{CDF}$ of sum-rate distribution

allocation and relay selection scheme significantly outperform the semi-random scheme.

Next, we evaluate the rate-energy trade-off of the proposed scheme. To do so, Fig. 5, placed at the top of next page, depicts the variation in the achieved end-user sum-rate with respect to the harvested power $\eta_{\ell}$ demanded by the destination nodes for different values of maximum transmitted power. Each point depicted in Fig. 5 corresponds to the sum-rate mean achieved after 1,000 experiment realizations. For the sake of illustration, we assume $\eta=\eta_{1}=\ldots=\eta_{L}$. Fig. 5(a) illustrates the effect of increasing the number of relays from $K=3$ to $K=$ 10 for a network composed of $L=2$ users and considering $N=6$ OFDM sub-carriers; while Fig. 5(b) focuses on the effect of increasing the number of sub-carriers from $N=16$ to $N=32$ for a network composed of $K=3$ relays and $L=2$ users. As expected, the rate-energy trade-off is evident as the sum-rate decreases when the demanded power increases. In addition, we can observe from Fig. 5 that increasing the number of relay nodes as well as the number of OFDM subcarriers provides more diversity to the system, which translates into better performance in terms of overall system sum-rate.

Finally, we examine the effect of increasing power demand on the assignment of PS ratios at the destination nodes, which is illustrated in Fig. 6. For this purpose, we consider the system setup of $K=3$ relay nodes and $L=2$ users, with $N=6$ OFDM sub-carriers. It is noteworthy that the PS ratios increase linearly in order to satisfy more stringent constraints on the demanded harvested power. As a consequence, the achievable sum-rate at the destination nodes reduces when increasing the power harvesting demands.

\section{CONCLUSION}

In this paper, we have proposed a novel resource allocation and relay selection scheme for cooperative multi-user multirelay OFDM networks with SWIPT capabilities at the endusers. By considering the equivalent channel gain given by the harmonic mean of the two channel hops, we proposed a low complexity scheme which can noticeably improve the system performance in comparison to a semi-random scheme. In our future work, we plan to extend this work by solving the 
IEEE $15^{\text {th }}$ Int. Symp. on Wireless Comm. Systems 2018 (ISWCS'18), Lisbon, Portugal

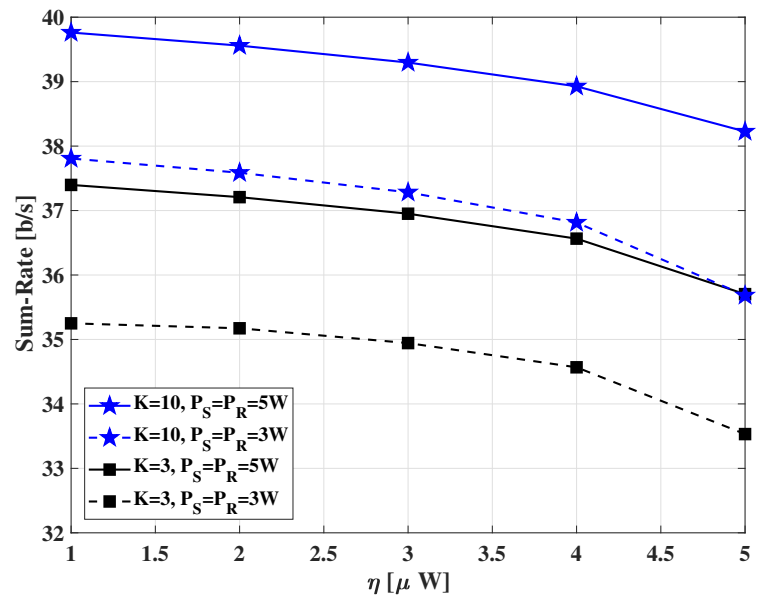

(a)

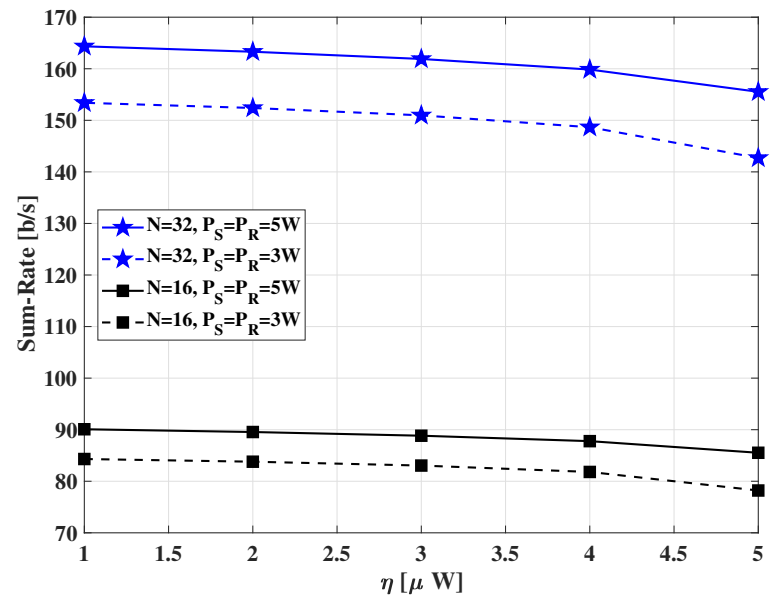

(b)

Fig. 5: Sum-rate versus demanded power: (a) $L=2$ and $N=6$; and (b) $K=3, L=2$.

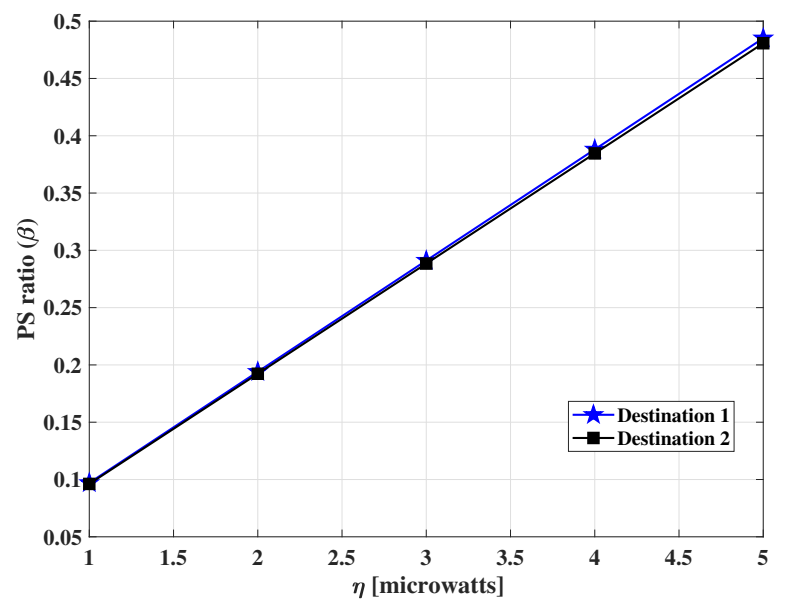

Fig. 6: Power splitting ratio versus demanded power

dual optimization problem of (P1) and show the asymptotic optimality of this solution when the number of sub-carriers $N$ is large enough.

\section{ACKNOWLEDGMENT}

This work was partially supported by the Luxembourg National Research Fund (FNR) in the framework of the FNRFNRS bilateral project "InWIP-NET : Integrated Wireless Information and Power Networks".

\section{REFERENCES}

[1] G. Li, Z. Xu, C. Xiong, C. Yang, S. Zhang, Y. Chen, and S. Xu, "EnergyEfficient Wireless Communications: Tutorial, Survey, and Open Issues," Wireless Commun., vol. 18, no. 6, pp. 28-35, Dec. 2011.

[2] H.S. Matthews et al., "Planning Energy-Efficient and Eco-Sustainable Telecommunications Networks," Bell Labs Tech. J., vol. 15, no. 1, pp. 215-236, Jun. 2010.

[3] L. Varshney, "Transporting Information and Energy Simultaneously," IEEE Int. Symp. Information Theory (ISIT), Toronto, Canada, pp. 1612 1616, Jul. 2008.

[4] X. Zhou, R. Zhang, and C.K. Ho, "Wireless Information and Power Transfer: Architecture Design and Rate-Energy Tradeoff," IEEE Trans. Commun., vol. 61, no. 11, pp. 4754-4767, Nov. 2013.

[5] I. Krikidis, S. Timotheou, S. Nikolaou, G. Zheng, D. Ng, and R. Schober, "Simultaneous Wireless Information and Power Transfer in Modern Communication Systems," IEEE Commun. Mag., vol. 52, no. 11, pp. 104-110, Nov. 2014.
[6] X. Lu, P. Wang, D. Niyato, D.I. Kim, and Z. Han, "Wireless Networks With RF Energy Harvesting: A Contemporary Survey," IEEE Commun. Surveys Tuts., vol. 17, no. 2, pp. 757-789, 2015.

[7] L. Liu, R. Zhang, and K.C. Chua, "Wireless Information and Power Transfer: A Dynamic Power Splitting Approach," IEEE Trans. Commun., vol. 61, no. 9, pp. 3990-4001, Sep. 2013.

[8] W. Zhuang and M. Ismail, "Cooperation in Wireless Communication Networks," IEEE Wireless Commun., vol. 19, no. 2, pp. 10-20, Apr. 2012.

[9] Z. Ding, S. Perlaza, I. Esnaola, and V.H. Poor, "Power Allocation Strategies in Energy Harvesting Wireless Cooperative Networks," IEEE Wireless Commun., vol. 13, no. 2, pp. 846-860, Feb. 2014.

[10] L. Liu, R. Zhang, and K. C. Chua, "Wireless information transfer with opportunistic energy harvesting," IEEE Transactions on Wireless Communications, vol. 12, no. 1, pp. 288-300, January 2013.

[11] 3GPP LTE, TS-25.101 v6.4.0, "Technical Specification Group RAN: User Equipment Radio Transmission and Reception," Mar. 2004.

[12] IEEE Std 802.16m-2011, "IEEE Standard for Local and Metropolitan Area Networks Part 16: Air Interface for Broadband Wireless Access Systems; Amendment 3: Advanced Air Interface,” Jun. 2011.

[13] Y. Li, W. Wang, J. Kong, and M. Peng, "Subcarrier Pairing for Amplifyand-Forward and Decode-and-Forward OFDM Relay Links," IEEE Commun. Lett., vol. 13, no. 4, pp. 209-211, Apr. 2009.

[14] I. Hammerstrom and A. Wittneben, "On the Optimal Power Allocation for Nonregenerative OFDM Relay Links," IEEE Int. Conf. on Communications, Istanbul, Turkey, 2006.

[15] A. Bletsas, A. Khisti, D.P. Reed, and A. Lippman, "A Simple Cooperative Diversity Method Based on Network Path Selection," IEEE J. Sel. Areas Commun., vol. 24, no. 3, pp. 659-672, Mar. 2006.

[16] M.O. Hasna and M.S. Alouini, "End-to-End Performance of Transmission Systems with Relays Over Rayleigh-Fading Channels," IEEE Trans. Wireless Commun., vol. 2, no. 6, pp. 1126-1131, Nov. 2003.

[17] W. Dang, M. Tao, H. Mu, and J. Huang, "Subcarrier-Pair Based Resource Allocation for Cooperative Multi-Relay OFDM Systems," IEEE Trans. Wireless Commun., vol. 9, no. 5, pp. 1640-1649, May 2010.

[18] S. Biyanwilage, U. Gunawardana, and R. Liyanapathirana, "Selective Sub-Carrier Relaying and Power Allocation for Multi-Relay-Assisted Cooperative OFDM Systems with Outdated CSI," Int. Conf. on Telecommunications (ICT), Ayia Napa, Cyprus, pp. 528-533, May. 2011.

[19] K. Xiong, P. Fan, C. Zhang, and K. Letaief, "Wireless Information and Energy Transfer for Two-Hop Non-Regenerative MIMO-OFDM Relay Networks," IEEE J. Sel. Area Commun., vol. 33, no. 8, pp. 1595-1611, Aug. 2015.

[20] Y. Liu and X. Wang, "Information and Energy Cooperation in OFDM Relaying: Protocols and Optimization," IEEE Trans. Veh. Technol., vol. 65, no. 7, pp. 5088-5098, Jul. 2016.

[21] S. Gautam, E. Lagunas, S.K. Sharma, S. Chatzinotas, and B. Ottersten, "Relay Selection Strategies for SWIPT-Enabled Cooperative Wireless Systems," IEEE Int. Symp. Personal, Indoor and Mobile Radio Communications (PIMRC), Montreal, Canada, Oct. 2017. 